\title{
Хроническая обструктивная болезнь легких у женщин
}

Кафедра госпитальной терапии № 1 лечебного факультета Московской медицинской академии им. И.М.Сеченова Росздрава: 119021, Москва, ул. Большая Пироговская, 6

\section{A.Tsvetkova, M.Kh.Mustafina \\ Chronic obstructive pulmonary disease in women}

Key words: COPD, epidemiology, women, gender, sex differences, tobacco smoke, air pollution.

Ключевые слова: хроническая обструктивная болезнь легких, эпидемиология, женщины, пол, половые различия, табакокурение, загрязнение воздуха.

\section{Эпидемиология хронической обструктивной болезни легких}

C начала XX в. в западных странах регистрировался активный рост табакокурения. Уже в середине XX в., когда > 1/2 населения Северной Америки курили, была известна связь рака легких и табакокурения. Однако к концу XX в. вследствие активной противотабачной политики государств в Северной Америке количество курильщиков уменьшилось. Аналогичной была ситуация и в европейских странах. Учитывая это, производители табачной продукции стали увеличивать производство и распространять продукцию в развивающихся странах. Так, в некоторых азиатских странах процент курящих мужчин составляет $>75 \%[1,2]$. Вскоре после снижения курения в Северной Америке уровень смертности от ишемической болезни сердца и инсульта снизился, однако уровень смертности от хронической обструктивной болезни легких (ХОБЛ) продолжал расти.

Какова же причина роста ХОБЛ в европейских странах и в Северной Америке при том, табакокурение стало гораздо мене активным? Наиболее значимым фактором может быть необратимость поражения легочной ткани вследствие табакокурения. То есть в дальнейшем, после отказа от курения, к уже сниженному уровню функционирования легких присоединяется возрастное снижение легочной функции. Это подтверждается предположением, что врачи-пульмонологи продолжают "пожинать плоды" табакокурения, имевшего место 10-20 лет назад. Возможно, в настоящее время женщины курят столь же активно, что и мужчины, однако являются более подверженными негативному воздействию табачного дыма. Развитие такой вредной привычки у более предрасположенного населения, возможно, увеличит распространенность ХОБЛ.

В середине XX в. смертность от ХОБЛ среди мужчин превышала смертность от ХОБЛ среди женщин в соотношении $2: 1$ или $3: 1$. В 1980 г. появились сообщения о росте заболеваемости ХОБЛ среди женщин и о выравнивании ее частоты. Канада была 1-й страной, которая заявила о преобладании женщин среди пациентов с ХОБЛ [3]. При сравнении по воз- растным категориям выяснилось, что женщины с большей вероятностью заболевают ХОБЛ в молодом и среднем возрасте. Только в старших возрастных группах преобладает мужская генерация. Ожидается, что уровень госпитализаций среди женщин возрастет к 2015 г. в 2 раза, как и уровень смертности [4] (рисунок).

Недавние исследования показали, что в период 1982-1996 гг. распространенность хронического бронхита возросла. Среди женщин она увеличилась на $65 \%$ (36,3 vs 59,8 на 1000 человек), среди мужчин на $50 \%$ (31,4 vs 47 на 1000 человек). Напротив, частота развития эмфиземы легких выше у мужчин по сравнению с женщинами. В 1997 г. в Великобритании распространенность ХОБЛ составляла 1,7\% среди мужчин и 1,4 \% - среди женщин. За период 1990-1997 гг. распространенность возросла на $25 \%$ у мужчин и на $69 \%$ - у женщин. Распространенность ХОБЛ среди мужчин находится на "плато" с середины 90-х гг. XX в., но растет среди женщин. Так, в 1997 г. частота ХОБЛ среди женщин достигла уровня, зарегистрированного среди мужчин в 1990 г. [5].

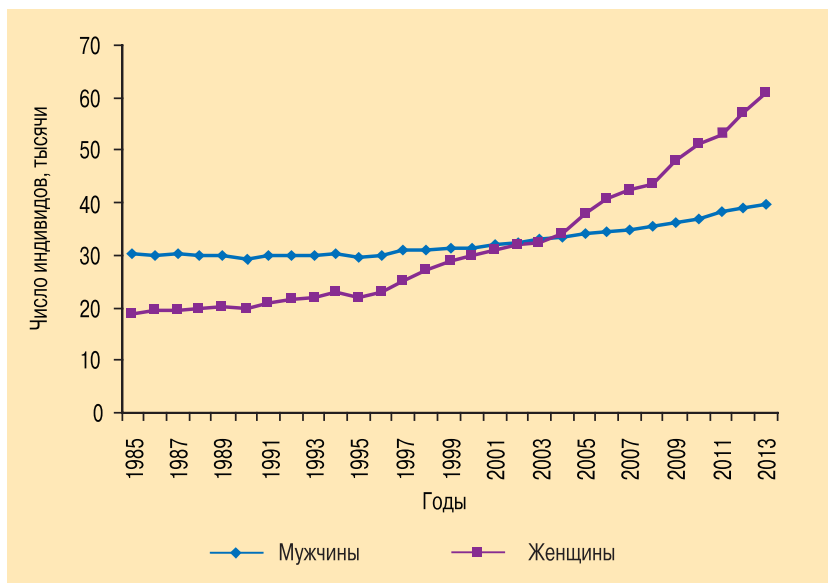

Рисунок. Настоящий (до 2000 г.) и предполагаемый (после 2000 г.) уровень госпитализаций больных мужчин и женщин с ХОБЛ в Канаде [4] 


\section{Причины развития ХОБЛ у женщин}

\section{Табакокурение}

В начале XX в. курение не было распространено среди женщин. Многими общественными организациями оно осуждалось как аморальное. Однако представители табачной индустрии пропагандировали образ курящей женщины - независимой, имеющей свободу выбора. В 1923 г. на долю женщин приходились только $5 \%$ от всех сигаретных продаж. Но уже в 1929 г. этот показатель возрос до $12 \%$, а в 1933 г. до $18,1 \%$. Пик потребления, 34 \% сигаретных продаж, был впервые зарегистрирован в 1965 г. С середины 80 -х гг. XX в. $\sim 25 \%$ женщин в США постоянно курят; процент курящих девушек постоянно растет [6].

В настоящее время в мире 200 млн курящих женщин: в развитых странах - 22\%, а в развивающихся - 9 \%. Ожидается, что доля куряших женшин в развивающихся странах к 2025 г. достигнет $20 \%$. Это означает, что к 2025 г. > 500 млн женщин в мире будут курить [7]. Известно, что у 15-20\% курильщиков развивается ХОБЛ, однако эта цифра может быть недооценена. Существует мнение, что женщины в большей степени предрасположены к повреждению легочной паренхимы по сравнению с мужчинами. Два перекрестных исследования с участием курильщиков в Пекине и Копенгагене показали, что у женщин более выражено нарушение легочной функции вследствие курения, чем у мужчин $[8,9]$.

В ранних исследованиях с участием курящих мужчин и женщин делался вывод, что женщины и мужчины в равной степени предрасположены к развитию ХОБЛ. Однако в этих исследованиях оценивалось развитие ХОБЛ по количеству выкуриваемых сигарет, но не по гендерному признаку. В более поздних исследованиях учитывались гендерновозрастные особенности. Так, в исследовании здоровья легких (Lung Health Study) предполагалось, что женщины более подвержены развитию ХОБЛ по сравнению с мужчинами. В исследовании принимали участие 5887 курящих мужчин и женщин в возрасте 35-59 лет. У курильщиков этого возраста отмечалось средней степени ограничение воздушного потока по дыхательным путям (объем форсированного выдоха за 1-ю с $\left(\mathrm{OФB}_{1}\right)$ - в пределах 5990 \%олж.). В этих исследованиях было продемонстрировано, что у женщин респираторный ответ на метахолин активнее [10]. Это может быть объяснено

Таблица 1

Тяжесть ХОБЛ у мужчин и женщин относительно длительности курения [11].

\begin{tabular}{|l|c|c|}
\hline \multirow{2}{*}{ Степень тяжести ХОБЛ } & \multicolumn{2}{|c|}{ Длительность курения, пачек / лет } \\
\cline { 1 - 3 } & $\begin{array}{c}\text { женщины } \\
\text { Легкая }\end{array}$ & мужчины \\
\hline Средняя & 35,1 & 60,1 \\
\hline Тяжелая & 54,8 & 65,9 \\
\hline
\end{tabular}

более медленным ростом и меньшим размером дыхательных путей. Сопротивляемость дыхательных путей в 4 раза больше зависела от радиуса дыхательных путей и при сравнении с мужчинами женщины находились выше на экспоненциальной кривой зависимости сопротивляемости дыхательных путей от калибра. Таким образом, при увеличении толщины слизистой бронхов женщины в большей степени могут страдать от обструкции по сравнению с мужчинами. Более крупные с возрастом дыхательные пути в меньшей степени склонны к критическому сужению.

Некоторые исследования показали больший риск возникновения дыхательной патологии у женщин вследствие курения, по сравнению с мужчинами. E. Prescott et al. [9] опубликовали результаты 2 больших европейских исследований по снижению легочной функции вследствие курения. В исследовании Copenhagen City Heart Study (CCHS) принимали участие 13897 мужчин и женщин, рожденных в 20-е гг. XX в. В исследовании Glostrup Population Study (GPS) принимали участие 4814 мужчин и женщин. Так, в CCHS скорость снижения легочной функции составляла 7,4 мл у женщин и 6,3 мл у мужчин. В GPS эти показатели равнялись 10,5 мл и 8,4 мл соответственно. R.Carter et al. [11] продемонстрировали схожие результаты в меньшей группе мужчин и женщин. Учитывая тяжесть заболевания по спирометрическим показателям и определяя длительность табакокурения, исследователи обнаружили, что женщины имели большую степень обструкции в зависимости от длительности приема и количества выкуриваемых сигарет. Результаты исследования могут быть объяснены склонностью женщин к недооценке табакокурения или переоценке такового у мужчин. Результаты этой работы полностью согласуются с эпидемиологическими данными по ХОБЛ и данными по другим табакоассоциированным заболеваниям, включая рак легких. Женщины при сравнении с мужчинами демонстрируют более выраженную обструкцию дыхательных путей в зависимости от длительности и количества выкуренных сигарет [11] (табл. 1).

\section{Загрязнение воздуха}

Помимо табакокурения, другим фактором риска развития ХОБЛ является загрязнение воздуха смесью отдельных частиц и капель жидкостей, различных по форме, размеру, составу и происхождению [12]. В развивающихся странах оно является большой проблемой. Мельчайшие частицы в воздухе приносят больший вред здоровью, поскольку при вдохе глубже проникают по дыхательным путям в легкие [13]. Чаще всего это продукты горения: сажа, конденсаты кислот, сульфаты или нитраты металлов [12]. В то время как загрязнение воздуха на открытом пространстве воздействуют на мужчин и женщин в равной степени, продукты горения в помещениях больше влияют на здоровье женщин. Это обусловлено применением необработанного топлива, такого как уголь, навоз, перегной, дерево, для обогрева помещений и приготовления пищи. 


\section{Инфекции}

Значительным фактором риска ХОБЛ являются инфекции. ВИЧ и туберкулез распространены по всему миру. Буллезная эмфизема была выявлена с помощью мультиспиральной компьютерной томографии у > 12 \% пациентов с ВИЧ [14]. Наличие ВИЧ у больного приводит к большей предрасположенности развития эмфиземы у курильщиков. Не ясно, приводит ли ВИЧ-инфекция самостоятельно или вторично к разрушению легочной паренхимы, однако в исследованиях с использованием бронхоальвеолярного лаважа было выявлено большое количество цитотоксичных лимфоцитов в легких у серо-позитивных по ВИЧ пациентов; также была выявлена корреляция концентрации этих лимфоцитов в легких и развития эмфиземы $[15,16]$. В отличие от ВИЧ-инфекции, туберкулез является давно известным инфекционным заболеванием. Известно, что ХОБЛ часто развивается на уже пораженных туберкулезом легких [17]. Женщины имеют особый риск развития как ВИЧ, так и туберкулеза. При этом среди женщин чаще имеет значение социокультурное влияние. У них в большей степени, по сравнению с мужчинами, существует страх стигматизации, вследствие этого - отсутствие обращения за медицинской помощью, нехватка времени и средств для лечения.

\section{Профессиональное воздействие}

Хорошо известны вредные вещества и химикаты, контакт с которыми может провоцировать развитие ХОБЛ. К ним относятся профессиональные загрязнители горной и тяжелой промышленности при добыче каменного угля, слюды, свинца, цинка, марганца, подрывных работах, измельчении песка, строительстве. Большое значение имеет воздействие на легочную ткань веществ, выделяемых при традиционно женских видах работ - в текстильной промышленности, при полировке драгоценных камней, в горном деле, керамическом и стекольном производстве. Известно, что длительное воздействие этих факторов может приводить к частично обратимой обструкции дыхательных путей. В качестве примера можно привести гендерно-ассоциированное заболевание биссиноз. Это поражение легочной паренхимы вследствие воздействия хлопковой пыли, похожее на багассоз (патология, развивающаяся под влиянием пыли тростникового сахара). Также биссиноз может возникать при длительном вдыхании пыли льна, конопли. В одном китайском исследовании было замечено, что в когорте китайских текстильных работников, более половины из которых - женщины, практически все не курили. Однако ежегодное снижение ОФВ 1 у них было больше, чем в контрольной группе, особенно у курильщиков [18].

\section{Генетические факторы}

Предполагается, что все вышеперечисленные факторы сами по себе не могут вызвать развитие ХОБЛ. Необходимо взаимодействие модифицирующих фак- торов с особенностями самого организма, часть которых в большей степени выражены у женщин. Хорошо известен генетический фактор - недостаточность $\alpha_{1}$-антитрипсина, что проявляется синдромом Марфана, синдромом Элерса-Данлоса [19]. S.Birring et al. [20] проспективно определили 25 пациентов ХОБЛ, которые не являлись курильщиками или имели индекс курения < 5 пачек / лет. 86 \% из этих пациентов с неясной причиной ХОБЛ (недостаточность $\alpha_{1}$-антитрипсина отсутствовала) были женщинами.

При наблюдении 44 семей с ранним развитием ХОБЛ наибольшая частота заболевания была выявлена среди женщин (79,6 \%) [21]. При исключении из группы настоящих и бывших курильщиков было обнаружено, что у женщин-родственниц I степени индекс Тиффно значительно ниже, а бронхиальная гиперреактивность - выше, чем у мужчин І степени родства. Более того, мультивариантный анализ этой же группы показал больший риск снижения ОФВ и большую бронхиальную чувствительность у женщин I степени родства, по сравнению с мужчинами [22].

\section{Другие причины}

Фактор риска ХОБЛ у женщин может быть связь с аутоиммунными механизмами. Так, в исследовании с участием 2 групп женщин оценивалась мокрота. В 1-й группе она содержала большое количество эозинофилов, во 2-й - нейтрофилов. Интересно, что во 2-й группе частота развития аутоиммунных заболеваний, в частности тиреотоксикоз, была выше [20]. Значение этого исследования окончательно не определено, однако существует предположение, что в некоторых случаях, особенно у некурящих, ХОБЛ формируется в следствие иммунной дисрегуляции, которая наиболее часто встречается у женщин.

\section{Потенциальные факторы, предрасполагающие к развитию ХОБЛ у женщин}

У женщин дыхательная патология может развиваться вследствие меньшего размера дыхательных путей. Эта физиологическая особенность вносит вклад в формирование заболевания и позволяет предположить, что женщины могут подвергаться большей дозе табачных продуктов по сравнению с мужчинами. Так, концентрация вредных веществ, получаемых женщиной при курении, оказывается больше, чем у мужчин, именно в силу меньшего размера легких. Однако различия дыхательных систем у женщин и мужчин не ограничивается размером.

Дыхательный размер легочной ткани увеличивается еще в течение 2 лет после рождения. Увеличение размера легких с возрастом является закономерным последствием увеличения дыхательной поверхности легких. При рождении легкие у девочек меньше, чем у мальчика, содержат меньшее количество бронхиол. Женские легкие созревают для выработки сурфактанта раньше, чем мужские. Сопротивляемость легочной ткани у новорожденных девочек обычно ниже, несмотря на меныший размер. Они меньше предрасположены 
к развитию респираторного дистресс-синдрома, чем мальчики, и в большей степени подвержены действию гормонов для продукции сурфактанта [23]. Женские легкие остаются меньшими по размерам, чем мужские, даже в пубертатный период, когда девочки крупнее мальчиков. Поскольку девочки обычно достигают своего максимального роста в позднем пубертате, их легочная паренхима также перестает увеличиваться, в то время как у мальчиков легкие могут расти вплоть до раннего взросления. У девочек и мальчиков рост легочной паренхимы и дыхательных путей находятся в диссонансе, однако наиболее это выражено у мальчиков. Конфигурация легких взрослой женщины - результат пропорционального роста ее дыхательных путей по отношению к легочной паренхиме. У мальчиков же рост дыхательных путей отстает от роста легочной паренхимы [24, 25].

Существует несколько объяснений различий развития обструкции дыхательных путей между мужчинами и женщинами. Большую роль играют гормональные или физиологические факторы у женщин, которые приводят к меньшей толерантности к сигаретному дыму. В частности, в исследовании на крыcax было показано, что при воздействии табачного дыма в течение 180 дней у самок отмечалось значительно повышение количества бокаловидных клеток в бронхах, по сравнению с самцами, что может свидетельствовать о большей предрасположенности женщин к развитию бронхита [26]. В исследованиях I.Tager et al., F.E.Speizer et al. было подтверждено, что в популяции риск развития ХОБЛ у женщин выше, чем у мужчин [27, 28].

Мужчины и женщины могут также иметь разные атопические проявления, которые выявляются кожными пробами или определением уровня IgE. Мальчики могут демонстрировать бо́льшую реактивность в раннем детстве, по сравнению с женщинами [29]. В одном скандинавском исследовании было показано, что у мужчин чаще отмечался повышенный уровень специфического для клещей IgE, однако этот иммуноглобулин не повышался при воздействии других домашних антигенов [30]. Изменение уровня специфического IgE может зависеть от таких факторов, как курение и профессиональные вредности.

Существуют доказательства того, что на состояние респираторной системы влияет гормональный фон.
Широко известно действие прогестерона, провоцирующего появление одышки у беременных. Изменения уровня прогестерона могут также провоцировать усиление астматических симптомов в зависимости от менструального цикла. В период до менструации и во время нее у женщин, не страдающих БА, снижался уровень выдыхаемого оксида азота [23]. Чувствительность дыхательных путей в течение менструального цикла не меняется. У женщин без БА повышаются плотность рецепторов к $\beta_{2}$-адреномиметикам и респираторная чувствительность к аденозинмонофосфату (АМФ) [31]. Экзогенный прогестерон, принимаемый женщинами в фолликулярной фазе менструального цикла, нарушает регуляцию плотности $\beta_{2}$-адренорецепторов. В исследованиях с участием женщин, больных БА, в лютеиновой фазе менструального цикла регистрировалось повышение плотности $\beta_{2}$-адренорецепторов. Экзогенное назначение прогестерона (не эстрогена) в фолликулярной фазе парадоксально снижает плотность $\beta_{2}$-адренорецепторов и чувствительность дыхательных путей к циклическому АМФ. Возрастной фактор также может влиять на эти сложные гормональные эффекты $\beta_{2}$-адренергической чувствительности [31].

В исследовании J.P.de Torres [32] был выявлен интересный факт: частота ХОБЛ не увеличивается у женщин старше 50 лет по сравнению с мужчинами, что, вероятно, обусловлено протективным эффектом менопаузы. Так, при сравнении 2 групп мужчинкурильщиков - моложе и старше 50 лет - частота ХОБЛ увеличивалась с 22 до $34 \%$, в то время как у женщин она оставалась постоянной (16\% vs $17 \%)$. Однако в этой работе не учитывались наступление менопаузы у женщин и использование ими заместительной гормональной терапии. Существует предположение о том, что заместительная гормональная терапия может препятствовать прогрессирующему снижению легочной функции [33]; в одном исследовании было показано, что заместительная гормональная терапия не повышает частоту вновь диагностированных больных ХОБЛ [34].

\section{Диагностические ошибки}

Известно, что "золотым стандартом" диагностики ХОБЛ является спирометрия. Однако в настоящее

Таблица 2

Предварительные диагнозы, установленные врачами при оценке гипотетического курильщика с одышкой [4]

\begin{tabular}{|c|c|c|c|}
\hline & \multicolumn{3}{|c|}{ Диагноз } \\
\hline & ХОБл, \% & БА, \% & Нереспираторное заболевание, \% \\
\hline \multicolumn{4}{|c|}{ Анамнез или физикальный осмотр } \\
\hline мужчины & 64,6 & 32,3 & 3,1 \\
\hline женщины & 49 & 43,8 & 7,3 \\
\hline \multicolumn{4}{|c|}{ Данные спирометрии } \\
\hline мужчины & 76 & 21,9 & 2,1 \\
\hline женщины & 64,6 & 32,3 & 3,1 \\
\hline \multicolumn{4}{|c|}{ Прием пероральных глюкокортикостероидов } \\
\hline мужчины & 85,4 & 10,4 & 4,2 \\
\hline женщины & 78,1 & 17,7 & 4,2 \\
\hline
\end{tabular}


время врачи по-прежнему не во всех случаях используют этот метод диагностики, и в связи с этим возрастает вероятность не распознать заболевание.

Помимо этого, у большинства врачей сложился определенный стереотип больного ХОБЛ: длительно курящий пожилой мужчина, предъявляющий жалобы на кашель, одышку. Примером является исследование с участием 192 врачей общей практики в Северной Америке [4]. При их опросе были обнаружены гендерные ошибки в диагностическом подходе к наиболее частым респираторным симптомам. В тех случаях, когда гипотетическим пациентом был мужчина, $2 / 3$ врачей рассматривали ХОБЛ как наиболее вероятный диагноз (табл. 2).

Однако в случаях, когда гипотетическим пациентом была женщина, < 1/2 врачей предполагали ХОБЛ. При выявленных с помощью спирометрии обструкции дыхательных путей и почти полном отсутствии ответа на бронходилататор частота диагноза ХОБЛ возросла и разница между полами уменьшилась. Когда же врачи установили, что лечение их пациентов глюкокортикостероидами не приносит результатов, диагноз ХОБЛ стал еще более вероятным, и разница между полами исчезла. К сожалению, только 1 врач из 5 в исследовании использовал спирометрию при первичной оценке вентиляционных нарушений курящего пациента.

Другая проблема состоит в том, что симптомы ХОБЛ рассматриваются как проявления БА. Путаница между БА и ХОБЛ - один из важнейших факторов в понимании эпидемиологии ХОБЛ. Среди детей БА чаще диагностируется у мальчиков, чем у девочек [35]. Некоторые исследователи полагают, что в действительности это заболевание в большей степени распространено среди девочек, однако они реже получают терапию по сравнению с мальчиками [36]. Среди подростков обоего пола уровень заболеваемости становится примерно одинаковым; среди взрослых женщины чаще лечатся от БА в стационаре [37]. Вероятно, они в большей степени склонны к обструктивным заболеваниям легких, чем мужчины, независимо от того, является ли эта обструкция обратимой. Также можно предположить, что у части женщин с БА на самом деле имеет место ХОБЛ. С другой стороны, существует и проблема гипердиагностики ХОБЛ. Так, например, легочный лимфангиолейомиоматоз часто расценивается как ХОБЛ [38, 39].

\section{Клиническое течение ХОБЛ у женщин}

Существует мало исследований по различиям клинического течения ХОБЛ у женщин и мужчин, однако имеются важные доказательства этих различий. Предполагают, что у женщин риск госпитализации по поводу обострений ХОБЛ больше, чем у мужчин: в исследованиях CCHS и GPS он был выше в 1,5-3,6 раза [9]. Этот факт согласуется с тяжестью течения заболевания у женщин, гендерными различиями в симптоматике. Тяжесть ХОБЛ у женщин обусловлена предрасположенностью к ее развития и более ранним формированием патологии, по сравнению с мужчинами. Однако доказательств большей смертности по поводу ХОБЛ среди женщин не существует. Предполагают, что женщины, страдающие ХОБЛ, чаще обращаются за медицинской помощью, по сравнению с мужчинами [37]. Эти различия могут быть социально обусловлены, однако существуют доказательства, что женщины и мужчины по-разному воспринимают респираторные симптомы и, видимо, поэтому женщины обращаются за медицинской помощью раньше.

В исследовании R.Carter et al. [11] были получены данные, позволяющие предположить, что у мужчин в большей степени снижается толерантность к физическим нагрузкам уже на ранних стадиях развития ХОБЛ, по сравнению с женщинами. Так, у мужчин с легкой обструкцией толерантность к физическим нагрузкам была на 33 \% меньше, чем в контрольной группе, тогда как у женщин с легкой обструкцией и здоровых показатели были идентичными. Однако у тех и других при нарастании обструкции дыхательных путей толерантность к физическим нагрузкам снижалась.

В этом исследовании было продемонстрировано, что мужчины, в отличие от женщин, теряют массу тела при прогрессировании заболевания, при сравнении с группой контроля. Эти изменения были определены по индексу массы тела, который снижался при увеличении обструкции дыхательных путей у мужчин, однако у женщин такие же значимые изменения отсутствовали. Этот факт можно объяснить несколькими причинами. Во-первых, повышение 24-часового расхода энергии вследствие нарушения механизма дыхания может вносить вклад в дополнительный расход калорий и приводить к уменьшению массы тела. Во-вторых, с увеличением дыхательной обструкции процесс приема пищи становится затруднительным, в частности, из-за усиления одышки. В-третьих, при прогрессировании заболевания может развиться анорексия. В-четвертых, вследствие различных факторов, как правило, в организме происходит замена мышечной ткани на жировую.

Отсутствие снижения общей массы тела у женщин связано с другими факторами: во-первых, особенностями течения заболевания; во-вторых, характерным пищевым поведением, отличным от мужчин. В-третьих, причиной отсутствия снижения веса у женщин могут быть неопределенные гормональные особенности. Наконец, мышечная масса изначально может быть низкой.

Данные этого исследования продемонстрировали, что в среднем анамнез курения у женщин меньше на 20 лет, по сравнению с мужчинами. При анализе обнаружилось, что у женщин показатель пачек / лет был меньше к тому моменту, когда значения легочной функции у них снизились до идентичных величин, зарегистрированных у мужчин, которые имели значительно более длительный анамнез курения. Длительность курения у мужчин коррелировала с тяжестью обструкции дыхательных путей, в то время как у женщин показатели были такими же при меньшем стаже курения. 
В нескольких публикациях исследуются различия во влиянии курения на мужчин и женщин. Во-первых, женщины действительно могут быть более восприимчивыми к сигаретному дыму, и обструкция у них развивается при меньшем воздействии табака, по сравнению с мужчинами. Во-вторых, анамнез курения у них может быть менее достоверным, т. к., согласно общественному мнению, курение часто является запретом для женщин, особенно для пожилых. В-третьих, женщины могут дополнительно страдать от пассивного курения. Если оно приводит к развитию легочных заболеваний, то воздействие табачных компонентов, особенно у женщин, может постоянно недооцениваться. В конце концов, сам процесс курения у мужчин и женщин различается: женщины склонны употреблять разные марки сигарет, которые могут в значительной степени влиять на здоровье [40]. Анализ эпидемиологических данных позволил предположить, что в действительности курение у женщин является таким же интенсивным, как и у мужчин.

\section{Особенности лечения ХОБЛ у женщин}

Женщины особым образом отвечают на терапию ХОБЛ, в которой большое место занимают профилактические мероприятия, например отмена курения для предупреждения дальнейшего прогрессирования заболевания. Уровень отмены курения низкий у пациентов обоих полов, однако, как было показано в многочисленных исследованиях, женщины бросают курить менее охотно. Так, по результатам Lung Health Study, женщины предпринимали меньшее количество попыток избавиться от курения, по сравнению с мужчинами [41]. Они реже применяют никотинозамещающую терапию (например, жевательную резинку) и чаще страдают от синдрома отмены. Один фактор поведения женщин был использован компаниями табачной продукции. Вследствие отмены курения часто увеличивается вес тела, и женщины страдают от этого в большей степени, чем мужчины [42]. Так, в Lung Health Study при отмене курения отмечался больший набор веса у женщин, по сравнению с мужчинами. В 1-й год после отмены курения у женщин масса тела увеличивалась в среднем на 5,2 кг vs 4,9 кг у мужчин), а затем - на 3,4 кг в год у женщин vs 2,6 кг у мужчин [43]. По окончании периода исследования у 19,1\% женщин и 7,6 \% мужчин массу тела повышалась на > $20 \%$ от первоначальной. Последствием стало снижение $\mathrm{OФВ}_{1}$ и ФЖЕЛ, более выраженное у женщин [44]. Кроме того, у женщин отмечалось более выраженное улучшение легочной функции при отмене курения по сравнению с мужчинами [45]. Производители табачной индустрии десятилетиями позиционировали сигареты как средство уменьшения веса. Когда в 20-х гг. XX в. в моду вошла худоба, специально для женщин был разработан бренд Virginia Slims.

Лечение ХОБЛ включает в себя применение бронходилататоров, холинолитиков, гормонов. Большинство этих препаратов назначаются в инга- ляционной форме, часто через небулайзеры. Исследования показали, что нарушение техники приводит к снижению и даже к отсутствию эффекта от терапии. По ряду предположений, женщины всех возрастов чаще по сравнению с мужчинами нарушают технику использования небулайзера, однако причины этого не ясны [46].

Кроме того, наиболее частым осложнением ХОБЛ у женщин является остеопороз [47].

\section{Частота ХОБЛ у женщин, по сравнению с мужчинами, при диагностике рака легких}

За последнее десятилетие был отмечен экспоненциальный рост заболеваемости и смертности от рака легких у женщин. Исследование уровня смертности в период 1990-1998 гг. выявило ежегодный прирост на $1,1 \%$ у женщин, по сравнению с 1,8 \% у мужчин. У женщин в 1987 г. заболеваемость раком легких превышала показатели по раку груди, и в 2003 г. его доля составила $25 \%$ среди всех онкологических заболеваний у женщин. Тесная связь между раком легких и обструктивными заболеваниями легких, такими как ХОБЛ, хорошо известна [49, 50].

Целью исследования S.Raghu Loganathan et al. [50] было сравнение частоты заболеваемости ХОБЛ с применением тестов исследования функции легких у мужчин и женщин при диагностике рака легких. Результаты показали, что при диагностике рака легких спирометрические признаки обструкции были выявлены у меньшего количества женщин, по сравнению с мужчинами. С учетом анамнеза курения и возраста было показано, что меньшую часть составили женщины, страдающие ХОБЛ. По этим данным можно предположить (но не доказать), что частота ХОБЛ у мужчин и женщин с раком легких различается.

Предположение, что связанные с табакокурением заболевания, такие как ХОБЛ и рак легких, характерны для мужчин, в настоящее время устаревает. Недавние данные, из US Surgeon General и Centers for Disease Control and Prevention (CDC) показали тенденцию увеличения уровня смертности от ХОБЛ у женщин [51-53].

Раком легких заболевают < 20 \% курильщиков, у такого же процента курящих развивается ХОБЛ. Однако > 85 \% пациентов с раком легких являются курильщиками и > 75 \% имеют спирометрические признаки ХОБЛ $[54,55]$. Несмотря на то, что курение является основным этиологическим фактором как ХОБЛ, так и рака легких, установлено, что обструкция дыхательных путей, выявленная с помощью спирометрии, является независимым фактором риска развития рака легких. Так, в некоторых исследованиях было отмечено 4-6-кратное возрастание этого риска, по сравнению с пациентами без дыхательной обструкции [48]. Однако точный механизм формирования рака легких вследствие ХОБЛ неизвестен.

В исследовании S.Lam et al. [56], в котором изучались изменения бронхиального эпителия с учетом 
статуса курения и пола, спирометрия была проведена у 401 пациента (212 мужчин и 189 женщин). Было обнаружено, что обструкция дыхательных путей (по критериям ATS [57]) у мужчин была в большей степени ассоциирована с преинвазивными бронхиальными повреждениями. Более того, женщины меньше курили (более низкий показатель пачек/ лет) и имели лучшую легочную функцию, по сравнению с мужчинами $(p=0,008)$.

\section{Заключение}

Частота ХОБЛ среди женщин стремительно растет и становится одной из ведущих причин женской смертности в мире [58]. На данный момент ХОБЛ у женщин встречается чаще, чем рак груди и легких. В то время как среди мужчин распространенность ХОБЛ достигла плато, в женской популяции она продолжает повышаться. Количество новых случаев заболеваемости ХОБЛ за год среди женщин увеличивается в 3 раза быстрее, чем у мужчин [59]. Глобально частота курящих мужчин достигла пика и медленно снижается, однако среди женщин курение получает все большую распространенность.

Для решения вопросов профилактики, диагностики и лечения ХОБЛ у женщин была созвана группа из 60 экспертов различных специальностей. Собрание состоялось при содействием ICEBERG. Возможно, этот симпозиум инициирует дальнейшие исследования, в которых будет продемонстрирована значимость проблемы заболевания ХОБЛ среди женщин.

\section{Литература}

1. Hu T.W., Tsai Y.W. Cigarette consumption in rural China: survey results from three provinces. Am. J. Publ. Hlth 2000; 90 (11): 1785-1787.

2. Kumra V., Markoff B.A. Who's smoking now? The epidemiology of tobacco use in the United States and abroad. Clin. Chest Med. 2000; 21 (1): 1-9.

3. McFarlane A., Goldstein R. COPD. In: Bryanton V., Chen Y., Johanson H. et al, eds. Respiratory disease in Canada. Ottawa: Health Canada; 2001. 45-55.

4. Chapman K.R., Tashin D.P., Pye D.J. Gender bias in the diagnosis of COPD. Chest 2001; 119 (6): 1691-1695.

5. Soriano J.R., Maier W.C., Egger P. et al. Recent trends in physician diagnosed COPD in women and men in the UK. Thorax 2000; 55: 789-794.

6. Gritz E.R. Cigarette smoking by adolescent females: implications for health and behavior. Women Hlth 1984; 9 (2-3): 103-115.

7. Mackay J., Amos A. Women and tobacco. Respirology 2003; 8: $123-130$

8. Xu X., Li B., Wang $L$. Gender difference in smoking effects on adult pulmonary function. Eur. Respir. J. 1994, 7: 477-483.

9. Prescott E., Bjerg A.M., Andersen P.K. et al. Gender difference in smoking effects on lung function and risk of hospitalization for COPD: results from a Danish longitudinal population study. Eur. Respir. J. 1997, 10: 822-827.

10. Kanner R.E., Connett J.E., Altose M.D. et al. Gender difference in airway hyperresponsiveness in smokers with mild COPD: The Lung Health Study. Am. J. Respir. Crit. Care Med. 1994; 150: 956-961.
11. Carter R., Nicotra B., Hurber G. Differing effects of airway obstruction on physical work capacity and ventilation in men and women with COPD. Chest 1994; 106 (6): 1730-1739.

12. Dockery D.W., Pope C.A., Xu X. et al. An association between air pollution and mortality in six US cities. N. Engl. J. Med. 1993; 329: 1753-1759.

13. Miller F.J., Gardner D.E., Graham J.A. et al. Size considerations for establishing a standard for inshalable particles. J. Air Pollut Control Assoc. 1979, 29: 610-615.

14. Diaz P.T., Clanton T.L., Pacht E.R. Emphysema-like pulmonary disease associated with human immunodeficiency virus infection. Ann. Intern. Med. 1992\$ 116: 124-128.

15. Diaz P.T., King E.R., Wewers M.D. et al. HIV infection increases susceptibility to smoking-induced emphysema. Chest 2000, 117 (5, suppl. 1): 285S.

16. Diaz P.T., King M.A., Pacht E.R. et al. Increased susceptibility to pulmonary emphysema among HIV-seropositive smokers. Ann. Intern. Med. 2000; 132: 369-372.

17. Snider G.L., Doctor L., Demas T.A. et al. Obstructive airway disease in patients with treated pulmonary tuberculosis. Am. Rev. Respir. Dis. 1971; 103: 625-640.

18. Christian D.C., Wang X. Respiratory effects of long-trem cotton dust. Curr. Opin. Pulm. Med. 2003, 9: 151-155.

19. Lee P., Gildea T.R., Stoller J.K. Emphysema in nonsmokers: alpha 1-antitrypsin deficiency and other causes. Cleveland Clin. J. Med. 2002; 69: 928-946.

20. Birring S.S., Brightling C.E., Bradding P. et al. Clinical, radiologic, and induced sputum features of chronic obstructive pulmonary disease in nonsmokers - a descriptive study. Am. J. Respir. Crit. Care Med. 2002; 166 (8): 1078-1083.

21. Silverman E.K., Chapman H.A., Drasen J.M. et al. Genetic epidemiology of severe, early-onset chronic obstructive pulmonary disease: risk to relatives for airflow obstruction and chronic bronchitis. Am. J. Respir. Crit. Care Med. 1998, 157: $1770-1778$.

22. Sliverman E.K., Weiass S.T., Drazen J.M. et al. Genderrelated differences in severe, early-onset chronic obstructive pulmonary disease. Am. J. respir Crit. Care Med. 2000, 162: 2152-2158.

23. Becklake M.R., Kauffmann F. Gender differences in airway behaviour over the human life span. Thorax 1999; 54 (12): 1119-1138.

24. Martin T.R., Castile R.G., Fredberg J.J. et al. Airway size is related to sex but not lung size in normal adults. J. Appl. Physiol. 1987; 63 (5): 2042-2047.

25. Mead J. Dysanapsis in normal lungs assessed by the relationship between maximal flow, static recoil, and vital capacity. Am. Rev. Respir. Dis. 1980; 121 (2): 339-342.

26. Hayashi M., Sornberger G.C., Huber G.L. Differential response in the male and female tracheal epithelium following exposure to tobacco smoke. Chest 1978; 73: 515-518.

27. Tager I., Tishler P.V., Rosner B. et al. Studies of the familial aggregation of chronic bronchitis and obstructive airways disease. Int. J. Epidemiol. 1978; 7: 55-62.

28. Speizer F.E., Rosner B., Tager I. Familial aggregation of chronic respiratory disease: use of a national health interview survey data for specific hypothesis testing. Int. J. Epidemiol. 1976; 5: 167-172.

29. Osman M. Theraputic implications of sex differences in astma and atopy. Arch. Dis. Child. 2003; 88 (7): 587-590.

30. Omenaas E., Bakke P., Elsayed S. et al. Total and specific serum IgE levels in adults: relationship to sex, age and environmental factors. Clin. Exp. Allergy 1994; 24 (6): 530-539.

31. Tan K.S., McFarlane L.C., Lipworth B.J. Paradoxical downregulation and desensitization of $\beta_{2}$-adrenoreceptors by 
exogenous progesterone in female asthmatics. Chest 1997; 111 (4): 847-851.

32. de Torres J.P., Campo A., Casanova C. Gender and chronic obstructive pulmonary disease in high-risk smokers. Respiration 2006; 73: 306-310.

33. Carlson C.L., Cushman M., Enright P.L. et al. Hormone replacement therapy is associated with higher $\mathrm{FEV}_{1}$ in elderly women. Am. J. Respir. Crit. Care Med. 2001; 163: 423-428.

34. Barr R.G., Wentowski C.C., Grodstein F. et al. Prospective study of postmenopausal hormone use and newly diagnosed asthma and chronic obstructive pulmonary disease. Arch. Intern. Med. 2004; 164: 379-386.

35. DeMarco R., Locatelli F., Suyer J., Burney P. European Community Respiratory Health Survey. Differenses in incidence of report asthma related to age in men and women. A retrospective analysis of the data of the European Respiratory Health Survey. Am. J. Respir. Crit. Care Med. 2000; 162 (1): 68-74.

36. Kuhni C.E., Sennhauser F.H. The Yentl syndrome in childhood astma: risk factors for undertreatment in Swiss children. Pediatr. Pulmonol. 1995; 19: 156-160.

37. Prescott E., Lange P., Vestbo J. et al. Effect of gender on hospital admissions for astma and prevalence of self-reported asthma: a prospective study based on a sample of the general population. Thorax 1997; 52 (3): 287-289.

38. Dahi H., Ie S.R., David O., Rubio E.R. Lymhangioleiomyomatosis: an unusial cause of dyspnea in young women. J. La St. Med. Soc. 2002; 154 (2): 78-81.

39. Urban T., Lazor R., Lacronique J. et al. Pulmonary lymhangioleiomyomatosis. A study of 69 patients. Groupe d'Etudes et de Recherche sur les maladies "Orphelines" Pulmonaires (GERM "O"P). Medicine (Baltimore) 1999; 78 (5): 321-337.

40. Huber G.L., Mahajan V.K., Rubin A.H. Theoretical and experimentally quantifiable determinants of tobacco smoking behavior for the development of successful smoking cessation strategies. Cancer Detect Prev. 1990; 14: 505-514.

41. Bjomson $W$., Rand C., Connet J.E. et al. Gender differences in smoking cessation after 3 years in the Lung Health Study. Am. J. Pub. Hlth 1995; 85: 223-230.

42. Gritz E.R., Crane L.A. Use of diet pills and amphetamines to lose weight among smoking and nonsmoking high school seniors. Hlth Psychol. 1991; 10 (5): 330-335.

43. O'Hara P., Connet J.E., Lee W.W. et al. Early and late weight gain following smoking seccation in the Lung Health Study. Am. J. Epidemiol. 1998; 148 (9): 821-830.

44. Wise R.A., Enright P.L., Connet J.E. et al. Effect of weight gain on pulmonary function after smoking cessation in the Lung Health Study. Am. J. Respir. Crit. Care Med. 1998; 157 (3, pt. 1): 866-872.

45. Connet J.E., Murray R.P., Buist A.S. et al. Changes in smoking status affect women more than men: results of the Lung Health Study. Am. J. Epidemiol. 2003; 157 (11): 973-979.

46. Goodman D.E., Israel E., Rosenberg M. et al. The influence of age, diagnosis and gender on proper use of metered dose inhalers. Am. J. Respir. Crit. Care Med. 1994; 150: $1256-1261$.
47. Iqbal F., Michaelson J., Thaler L. et al. Declining bone mass in men with chronic pulmonary disease - contribution of glucocorticoid treatment, body mass index, and gonadal function. Chest 1999; 116 (6): 1616-1624.

48. Skillrud D.M., Offord K.P., Miller R.D. Higher risk of lung cancer in COPD: a prospective randomized matched controlled study. Ann. Intern. Med. 1986; 105: 503-550.

49. Tockman M.S., Anthonisen N.R., Wright E.C. et al. Airways obstruction and the risk for lung cancer. Ann. Intern. Med. 1987; 106: 512-518.

50. Loganathan R.S., Stover D.E., Weiji S., Ennapadam V. Prevalence of COPD in women compared to men around the time of diagnosis of primary lung cancer. Chest 2006; 129: 1305-1312.

51. Centers for Disease Control and Prevention. Recent trends in mortality rates for four major cancers, by sex and race / ethnicity: United States, 1990 to 1998. J. A. M. A. 2002; 287: 1391-1392.

52. US Department of Health and Human Services. The health consequences of smoking for women: a report of the Surgeon General. Washington, DC: US Department of Health and Human Services; 1980.

53. Women and smoking. A report of the US Surgeon General. Washington, DC: U.S. Department of Health and Human Services; 2001.

54. Lopez-Encuentra A. Bronchogenic Carcinoma Co-operative Group. Comorbidity in operable lung cancer: a multicenter descriptive study of 2992 patients. Lung Cancer 2002; 35: 263-269.

55. Congleton J., Muers M.F. Incidence of airflow obstruction in bronchial carcinoma, its relation to breathlessness and response to bronchodilator therapy. Respir. Med. 1995; 89: 291-296.

56. Lam S., LeRiche J.C., Zheng Y. et al. Sex-related differences in bronchial epithelial changes associated with tobacco smoking. J. Natl. Cancer Inst. 1999; 91: 691-696.

57. American Thoracic Society. Standardization of spirometry: 1994 update. Am. J. Respir. Crit. Care Med. 1995; 152: 1107-1136.

58. Brett K.M., Hayes S.G. Women's health and mortality chartbook. Washington, DC: DHHS Office on Women's Health; 2004.

59. Mannino D.M., Homa D.M., Akinbami L.J. et al. Chronic obstructive pulmonary disease surveillance: United States, 1971-2000. Morbid. Mortal. Wkly Rep. Surveill. Summ. 2002; 51: 1-16.

\section{Информация об авторах}

Цветкова Ольга Александровна - д. м. н., проф. кафедры госпитальной терапии № 1 лечебного факультета ММА им. И.М.Сеченова; тел.: (499) 248-73-12; e-mail: oatsvetkova@mail.ru

Мустафина Малика Харисовна - ординатор кафедры госпитальной терапии № 1 лечебного факультета ММА им. И.М.Сеченова; тел.: (499) 248-73-12; e-mail: malika_mstf@mail.ru 